\title{
Harmonic mode locking and multiple pulsing in a soft-aperture Kerr-lens mode-locked Ti:sapphire laser
}

\author{
Ja-Hon Lin ${ }^{\mathrm{a}}$, Wen-Feng Hsieh ${ }^{\mathrm{a}, *}$, Hsiao-Hua $\mathrm{Wu}^{\mathrm{b}}$ \\ a Institute of Electro-Optical Engineering, National Chiao-Tung University, 1001 Ta-Hsueh Rd., Hsinchu 300, Taiwan \\ ${ }^{\mathrm{b}}$ Department of Physics, Tunghai University, 181 Sec. 3 Chung Kang Road, Taichung 407, Taiwan
}

Received 11 April 2002; received in revised form 11 April 2002; accepted 20 August 2002

\begin{abstract}
We report the observation of harmonic mode locking and multiple pulse operations in a soft-aperture Kerr-lens mode-locked Ti:sapphire laser by varying the total intra-cavity dispersion. Second, third, and fourth order harmonic mode locking as well as multiple pulsing with interpulse separation ranging from femtosecond to nanosecond are observed. The laser is characterized in these regimes in terms of wavelength, bandwidth, pulsewidth, average and peak powers as functions of group velocity dispersion. By introducing a loss difference term into gain dynamic analysis, we conclude that gain depletion and recovery mechanisms are responsible for the underlying physics of current observations.
\end{abstract}

(c) 2002 Elsevier Science B.V. All rights reserved.

\section{Introduction}

Resulting from the soliton-like pulse shaping effect via the interplay between the self phase modulation (SPM) and the group velocity dispersion (GVD), generation of ultra-short pulses have been achieved in Kerr-lens mode-locked (KLM) Ti:sapphire lasers [1-4]. Because of extremely high nonlinear effects, it is not surprising that the pulsesplitting and multiple pulse operation can be observed in these laser systems besides the normal

\footnotetext{
${ }^{*}$ Corresponding author. Tel.: +886-3-5712121; fax: +886-35716631.

E-mail addresses: jhl.eo86g@nctu.edu.tw (J.-H. Lin), wfhsieh@mail.nctu.edu.tw (W.-F. Hsieh).
}

mode locking (NML). A single pulse per round trip splits into two or three pulses with fs spacing had been observed in a soft-aperture KLM (SAKLM) Ti:sapphire laser [2]. The pulse-splitting is attributed to side-lobe pulse separation by the interplay of the negative GVD and SPM in the laser cavity.

In a hard-aperture KLM (HAKLM) Ti:sapphire laser, other than the fs-split pulses, multiple pulse operations with asymmetric ns-splitting were also observed [3]. It is explained by the enhanced nonlinear interaction caused by two counterpropagating pulses coinciding within the active medium to stabilize the interpulse spacing equal to the round trip time between the active medium and one of the output couplers. With an ion-implanted semiconductor as the saturable-absorber mirror 
[4], double to quadruple pulses were observed in a passive mode-locked Ti:sapphire laser. The interpulse spacing was obtained ranging from several hundred fs to several ns without reliably reproducible spacing [4] when the GVD was decreased below a critical value. The results were directly compared with the simulation from the GinzburgLandau equation.

Recently, harmonic mode locking generated in the passive mode-locked fiber ring lasers were reported and attributed to soliton interaction by means of acoustic effects [5-7]. Harmonic operation of a passively mode-locked short-cavity $\mathrm{Cr}^{4+}$ :YAG laser with one, two, and three pulses in the cavity has been demonstrated [8] for various values of total cavity GVD. By increasing the intracavity power of a passively harmonic modelocked erbium/ytterbium fiber laser with short cavity, up to 11 pulses in the cavity with nearly equal pulse-to-pulse temporal spacing has also been observed [9]. An analytic model based on the interaction between the pulses through the transient gain depletion and recovery dynamics in the gain medium [10] was proposed to explain these phenomena. Similar gain dynamics had already been successfully applied to explain harmonic mode locking in an external cavity semiconductor laser by adjusting the injection current [11]. It contains two-section multiple quantum well structures of which one providing gain and the other acting as the slow saturable absorber.

We report for the first time to our knowledge the observation of second, third, and fourth order harmonic mode locking in a SAKLM Ti:sapphire laser without saturable absorber. Multiple pulsing with interpulse separation ranging from femtosecond to nanosecond was also observed. The mode-locked states are distinguished into three regions and characterized in terms of wavelength, time bandwidth product (TBP), pulse width, average and peak powers as function of group velocity dispersion. By introducing a loss difference of the split pulses into theoretical analysis of gain depletion and recovery model [10], we find that multiple pulses with unequal spacing between the pulses can take place in addition to the harmonic mode locking which occurs when the loss difference is neglected.

\section{Experiment}

The experiment was carried out by a SAKLM Ti:sapphire laser with a near symmetric cavity as shown in Fig. 1. The SAKLM Ti:sapphire laser consists of a 9-mm long Brewster-cut Ti:sapphire $\operatorname{rod}(\mathrm{Ti}: 0.1 \%, \mathrm{FOM}>150)$, two curved mirrors $\left(\mathrm{M}_{1}\right.$ and $\mathrm{M}_{2}$ ) of $10-\mathrm{cm}$ radius of curvature tilted by $10^{\circ}$ angle, and two flat mirrors - a 99\% high reflector $\mathbf{M}_{4}$ and a $95 \%$ output coupler $\mathbf{M}_{3}$. Both the distances between $\mathbf{M}_{2}$ and $\mathbf{M}_{4}$ as well as $\mathbf{M}_{1}$ and $\mathbf{M}_{3}$ are adjusted around $74 \mathrm{~cm}$ to form a near symmetric configuration. A pair of Brewster-angled SF10 prisms separated by $27 \mathrm{~cm}$ is inserted between $M_{1}$ and $M_{3}$ for dispersion compensation that allows for femtosecond pulses generation. The prism pair is mounted on the translation stage to finely adjust negative GVD. The negative GVD $\left(\beta_{2} z\right)$ can be calculated by the following expression [12]:

$$
\begin{aligned}
\beta_{2} z= & -\frac{2 \lambda^{3}}{\pi c^{2}}\left(\frac{\mathrm{d} n}{\mathrm{~d} \lambda}\right)^{2} L_{p}+\frac{\lambda^{3}}{\pi c^{2}}\left[\frac{\mathrm{d}^{2} n}{\mathrm{~d} \lambda^{2}}+\left(2 n-\frac{1}{n^{3}}\right)\right. \\
& \left.\times\left(\frac{\mathrm{d} n}{\mathrm{~d} \lambda}\right)^{2}\right] \frac{4 \sin (\varepsilon / 2)}{\left[1-n^{2} \sin ^{2}(\varepsilon / 2)\right]^{1 / 2}} \frac{\mathrm{d} n}{\mathrm{~d} \lambda} \Delta \lambda L_{p} \\
& +\frac{\lambda^{3}}{\pi c^{2}} \frac{\mathrm{d}^{2} n}{\mathrm{~d} \lambda^{2}} \tan (\varepsilon / 2)\left(d_{p 1}+d_{p 2}\right),
\end{aligned}
$$

where $z$ is single path length of the pulse propagation in cavity, $n$ and $\varepsilon$ are the refractive index and the apex angle of the prisms, $c$ is the speed of light in vacuum, $\lambda$ is the center wavelength, $\Delta \lambda$ is the optical-spectrum bandwidth, $L_{\mathrm{p}}$ is the prism

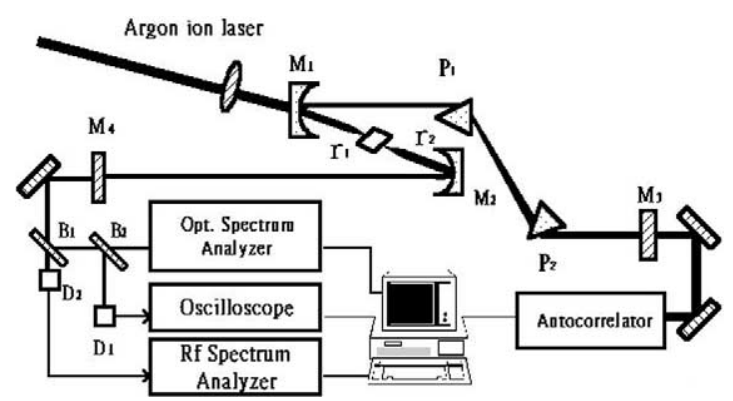

Fig. 1. Experimental setup of the Kerr-lens mode-locking Ti:sapphire laser. $B_{1}, B_{2}$ are beam splitter and $D_{1}, D_{2}$ are the high speed detectors. 
separation, and $d_{\mathrm{p} 1}$ as well as $d_{\mathrm{p} 2}$ are the insertion width of prisms $P_{1}$ and $P_{2}$, respectively. Thus the total intra-cavity GVD $\left(\beta^{\prime \prime}\right)$ could be estimated by taking the positive dispersion of the crystal into account. The total cavity length is approximate $160 \mathrm{~cm}$ that corresponds to the repetition rate of 93.3 MHz or round trip time of $10.7 \mathrm{~ns}$. An all-line continuous wave (CW) Ar ion laser (Coherent, Innova 300) was employed to pump the Ti:sapphire crystal via a plano-convex lens with the focal length of $12.7 \mathrm{~cm}$. Two fast photodiodes (ElectroPhysics Technology ET-2000 with rise time 100 ps) together with an oscilloscope (LeCroy 94504A, bandwidth $300 \mathrm{MHz}$ ) and an rf-spectrum analyzer (HP-8560E) are employed to observe the pulse train and its corresponding power-spectrum. A collinear autocorrelator combined with a GaAsP photodiode (Hamamtsu G117) acting as two-photon absorption detector [13] is constructed to achieve interferometric autocorrelation measurement of pulse waveforms. Finally, the optical-spectra were measured by using an opticalspectrum analyzer (Ophir wavestar).

\section{Results and discussion}

\subsection{Regimes for normal mode locking, harmonic mode locking, and multiple pulsing}

As our previous experimental results [14], the SAKLM operation usually occurs at the fractional degenerate cavity configurations [15] with peculiar mode patterns consisting of superposition of the fundamental mode with low-order degenerate transverse modes. In this experiment, we translated the curved mirror $\mathrm{M}_{2}$ along the optic axis of cavity to search for pertinent cavity configuration so that the SAKLM operation was achieved. Throughout the experiment, the pumping power and the insertion width of the first prism $\mathrm{P}_{1}$ into laser beam were kept to the value of $4.5 \mathrm{~W}$ and $0.37 \mathrm{~mm}$. Subsequently, we moved the translation stage to change the insertion width of the second prism $\mathrm{P}_{2}$ into laser beam in order to change the negative GVD. Besides the NML, the laser could also be operated with second, third, and fourth order harmonic mode locking as well as stable multiple pulsing whose interpulse separation ranges from femtosecond to nanosecond.

On the basis of our observation, we divided mode-locked operation into three regions as a function of the total intra-cavity GVD $\left(\beta^{\prime \prime}\right)$ that is shown later in Figs. 6 and 7. In region I, $\beta^{\prime \prime}$ is less than $-2250 \mathrm{fs}^{2}$ where only the NML is observed. Further increasing $\beta^{\prime \prime}$ to the region II $\left(-2250 \mathrm{fs}^{2}\right.$ $\leqslant \beta^{\prime \prime} \leqslant-1950 \mathrm{fs}^{2}$ ), the system exhibits two possible states either in the NML or in second harmonic mode locking (SHML) whose repetition period becomes one-half of the cavity round trip time. Occasionally, with a certain $\beta^{\prime \prime}$ in this region, unstable pulse-splitting with ps spacing is found that would slowly turn into the SHML. In region III of which $\beta^{\prime \prime}>-1950 \mathrm{fs}^{2}$, multiple pulses with unequal nanosecond interpulse spacing were observed and sometimes third harmonic mode locking (THML) or even fourth harmonic mode locking (FHML) pulses could be observed at the far limit of this region. Mostly, multiple pulses with unequal nanosecond interpulse spacing were found immediately as laser operated in this region. However, the NML may also be found initially and maintained in few-seconds to minutes and eventually developed into stable multiple pulse operation. Therefore, fewer data of the NML can be recorded in region III of Figs. 6 and 7 before they developed into pulse-splitting. We can also block the laser to destroy the mode locking at multiple pulsing and initial the mode locking again for observing the NML.

In Fig. 2, we show a typical interference autocorrelation trace (solid line) for the NML measured at $\beta^{\prime \prime} \approx-2768 \mathrm{fs}^{2}$ about $60 \mathrm{fs}$ pulsewidth that is fitted with the $\operatorname{sech}^{2}\left(-1.76 t / t_{0}\right)$ curve (dot line). The inset is the corresponding optical-spectrum with center wavelength at $825 \mathrm{~nm}$ and bandwidth of $12.4 \mathrm{~nm}$ by fitting with a $\mathrm{sech}^{2}$ profile (bold line). The autocorrelator can also be employed to resolve subpicosecond pulse-splitting. In Figs. 3(a) and (b), the autocorrelation trace exhibits pulse-to-pulse separation of 210 and 475 fs, respectively. As mentioned before [16,17], the phase difference between two pulses can be gained from the optical-spectrum with the relation $\varphi=2 \pi\left(\omega_{0}-\omega_{1}\right) /\left(\omega_{2}-\omega_{1}\right)$. Here $\omega_{0}$ is the central frequency of the fitting sech ${ }^{2}$ curve and $\omega_{1}$ and $\omega_{2}$ 


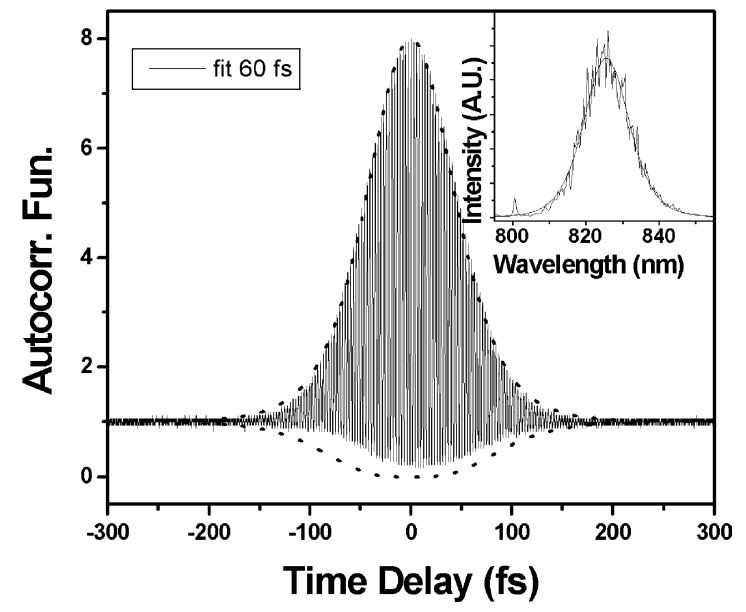

Fig. 2. Typical autocorrelation trace (solid curve) and corresponding optical-spectrum (inset) for the NML. The dash curve is fitted with the $\operatorname{sech}^{2}$ function.

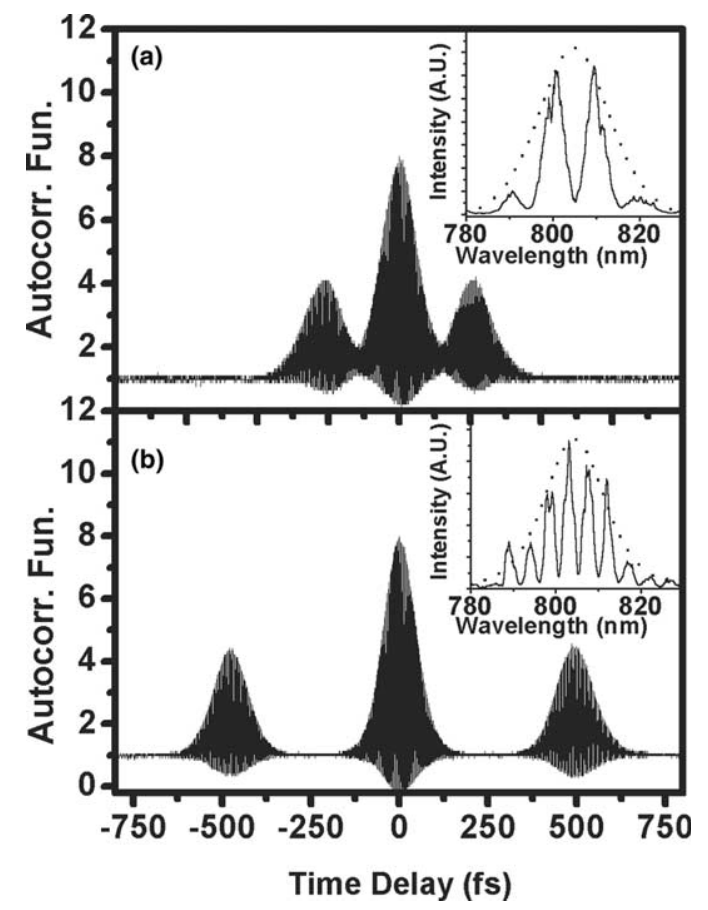

Fig. 3. Autocorrelation trace and corresponding optical-spectrum (inset) for double pulses with 210 separation as well as the relative phase $\approx \pi$ between two pulses (a), and $475 \mathrm{fs}$ splitting in conjunction with $\pi / 2$ phase difference in (b).

are the closest frequency to $\omega_{0}$. It reveals that the relative phases of the split pulses in Fig. 3(a) and (b) are $\pi$ and $\pi / 2$, respectively. Under different operation conditions, the change of relative phase of pulses around $\pi$ and 0 between pulses was also seen.

We have observed multiple pulses with the interpulse separation larger than 10 ps which could not be observed by our autocorrelator but could be displayed by the oscilloscope. The double pulses in Fig. 4(a) shows split spacing of 2.6 ns with its corresponding rf-spectrum in Fig. 4(b). Similarly, we observed stable three asymmetric ns-spacing pulses as shown in Fig. 4(c) whose rf-spectrum is in Fig. 4(d). In addition, the four pulses having pulse spacing 1.8, 1.3, and $2.6 \mathrm{~ns}$ followed by a 5 -ns blanking are shown in Fig. 4(e) with corresponding spectrum in Fig. 4(f). It is worth to mention that the relative spacing and amplitude for multiple pulses could be altered at the different total intra-cavity GVD while we change the insertion length of the second prism.

The pulse trains together with their corresponding rf-spectra for the NML, SHML, THML, and FHML are shown in Fig. 5, respectively. The period of pulse train for the NML shown in Fig. 5(a) is equal to cavity round trip time with four harmonics of longitudinal beating in corresponding rf-spectrum [see Fig. 5(b)]. In Fig. 5(c), the SHML state has two pulses within a round trip in which its spectrum shows the first and third harmonics being suppressed below the noise level ( -70 $\mathrm{dB}$ ) in Fig. 5(d) to verify that the pulse train is highly periodic. Similarly, highly periodic and suppressing of the first, second and fourth harmonics below the noise level in Figs. 5(e)-(f) displays the laser is at the THML state. The FHML appears in the system with only the fourth harmonic as shown in Fig. 5(h). However, due to the limited bandwidth of our $300-\mathrm{MHz}$ oscilloscope which is smaller than the $373-\mathrm{MHz}$ repetition rate of the FHML state, large pulse-to-pulse fluctuation is recorded in Fig. 5(g).

The variations of the measured average output power (solid symbols), pulsewidth (open symbols), and the intra-cavity peak power versus the total intra-cavity GVD $\left(\beta^{\prime \prime}\right)$ for the NML, SHML and double pulses are shown in Figs. 6(a) and (b). In Fig. 6(a) the average power (solid squares) and pulsewidth (open squares) of the NML are about $271 \mathrm{~mW}$ and $84 \mathrm{fs}$, respectively, at the largest $\left|\beta^{\prime \prime}\right|$ 


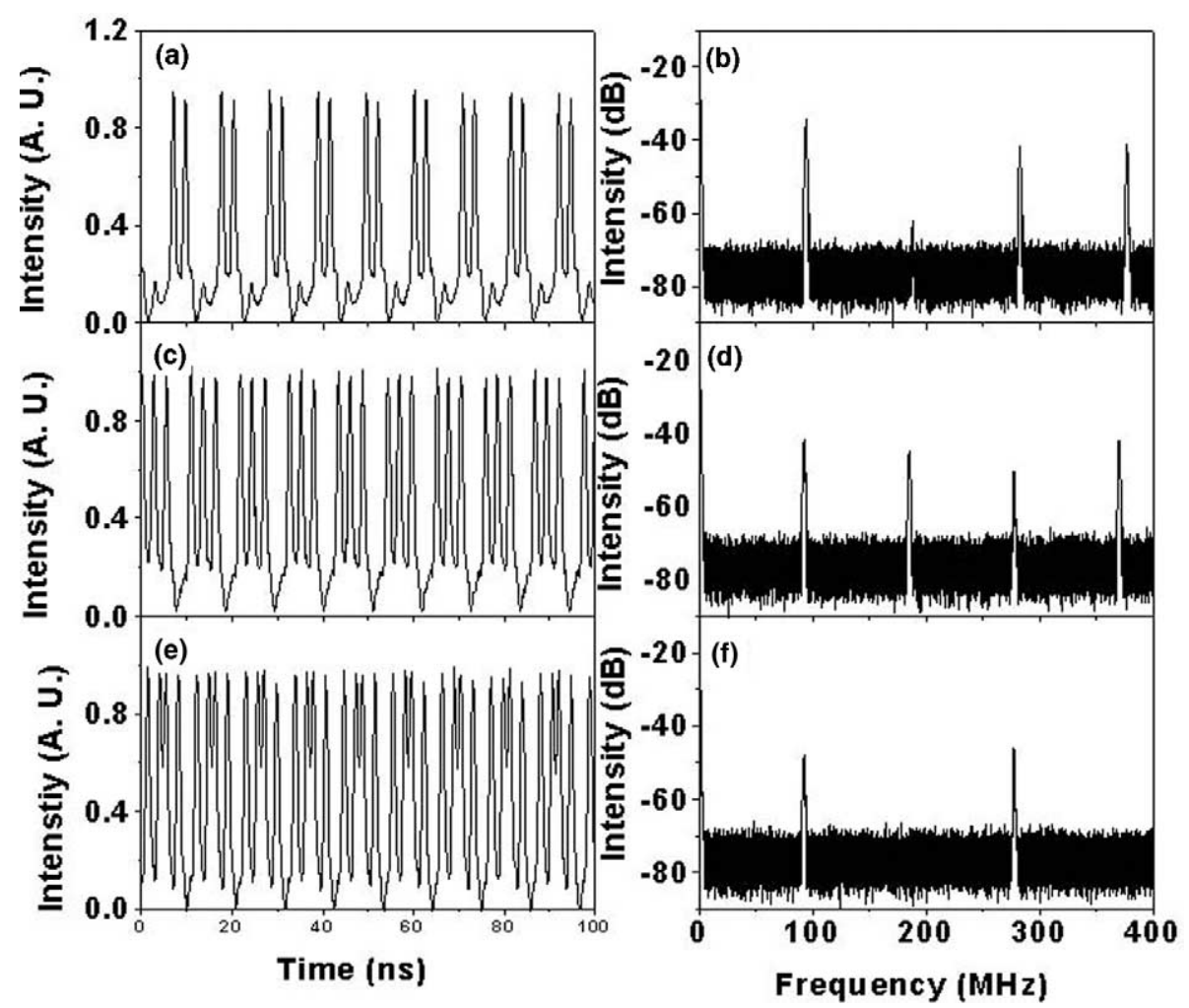

Fig. 4. Pulse trains for double (a), triple (c), and quadruple (e) pulses with asymmetric nanosecond separations and their corresponding rf-spectra (b), (d), and (f).

and then suddenly decrease to around $200 \mathrm{~mW}$ and $55 \mathrm{fs}$ at $\beta^{\prime \prime} \approx-2760 \mathrm{fs}^{2}$ in region I. Pulsewidth decrease accompanied with intra-cavity peak power increase [shown as solid squares in Fig. 6(b)] is due to better GVD compensation by the prism pair. In addition, the operation wavelength is continuously red shifted to the maximum excursion at $827 \mathrm{~nm}$ where the time bandwidth product (TBP) is near the transform-limit of 0.318 for $\beta^{\prime \prime} \approx-2760 \mathrm{fs}^{2}$ as shown in Fig. 7. Then the average power of the NML keeps almost constant until region III with a relatively small deviation of $10 \mathrm{~mW}$ or $5 \%$ and the pulsewidth is approximately $53 \mathrm{fs}$.

Since the 5.35 ns-separated pulses in region II can extract more energy from the active medium in a round trip, the SHML pulse (solid circle in Fig. 6(a)) shows higher average output power about $310 \mathrm{~mW}$ with $62 \mathrm{fs}$ pulsewidth at $\beta_{2}^{\prime \prime}=-2220 \mathrm{fs}^{2}$. However, the fact of two pulses in one round trip together with the broader pulsewidth for the SHML in comparison with the NML causes the pulses at the SHML (solid circle) in Fig. 6(b) having smaller intra-cavity peak power than that at the NML (solid square). Thus the peak power of single pulse at the SHML declines to $0.54 \mathrm{MW}$ (almost a half of the NML one). Generally, pulses at the NML state have a little higher value of the TBP near 0.39 and recover at the state of the SHML as shown in Fig. 7.

In region III of Figs. 6 and 7, two kinds of double pulses are found in our laser system and labeled with triangle and diamond symbols. One (triangle symbol) shows near equal spacing between pulses on the oscilloscope like that observe in the SHML. However, its odd number of longitudinal beatings on rf-spectrum are still above the noise level $(-70 \mathrm{~dB})$ revealing a little unequal interpulse spacing between pulses. The others with asymmetric nanosecond splitting spacing between 

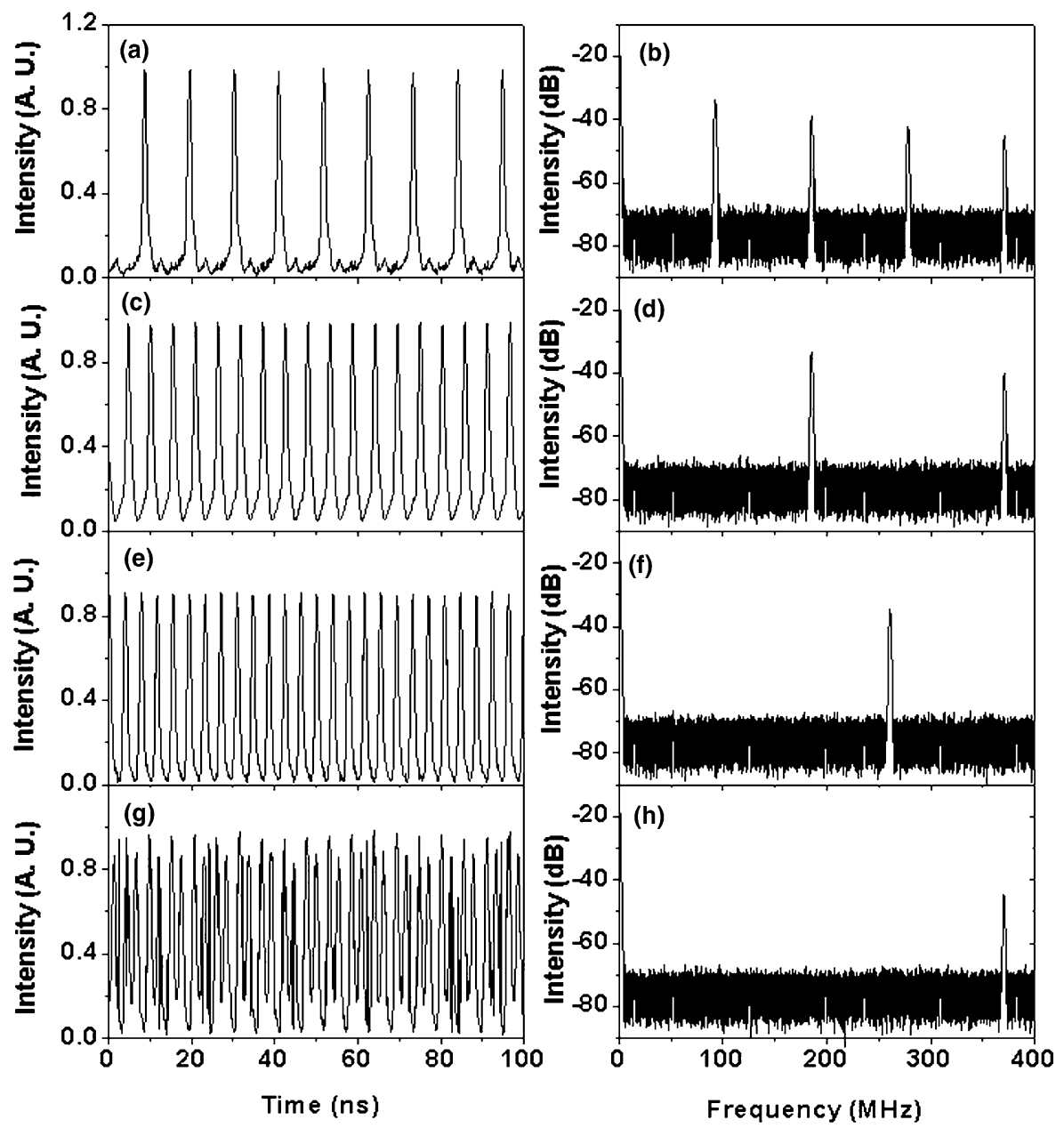

Fig. 5. Pulse trains for the NML (a), SHML (c), THML (e), and FHML (g) and their corresponding rf-spectra (b), (d), (f), and (h).

pulses are labeled diamond symbol whose time traces are shown in Fig. 4(a). Double pulses in region III of Fig. 6(a) reveals a little shorter pulsewidth than the SHML pulses in region II, but their bandwidth become a little broader to cause the value of the TBP slightly larger than the SHML in Fig. 7. In addition, the average power of double pulses in Fig. 6(a) tends to drop as $\left|\beta^{\prime \prime}\right|$ declining in region III to cause the peak power fall in Fig. 6(b). The operation wavelength of the pulses gradually decreases throughout the region II and III in Fig. 7 and the lasing wavelength at either the state of the SHML or double pulses is longer than that at the NML state.
3.2. Explanations for harmonic mode locking and multiple pulsing

Pulse-splitting with regular interspacing or harmonic mode locking has been observed in passive mode-locked fiber laser that was explained by the acoustic effect created by the electrostriction in fiber [5-7]. The refractive index change caused by the acoustic effect for a 9-mm Ti:sapphire crystal is too small to cause harmonic mode locking in such a laser. We therefore try to use the transient depletion and recovery of gain dynamics [10] which has been successfully applied to the passive harmonic mode-locked semiconductor 


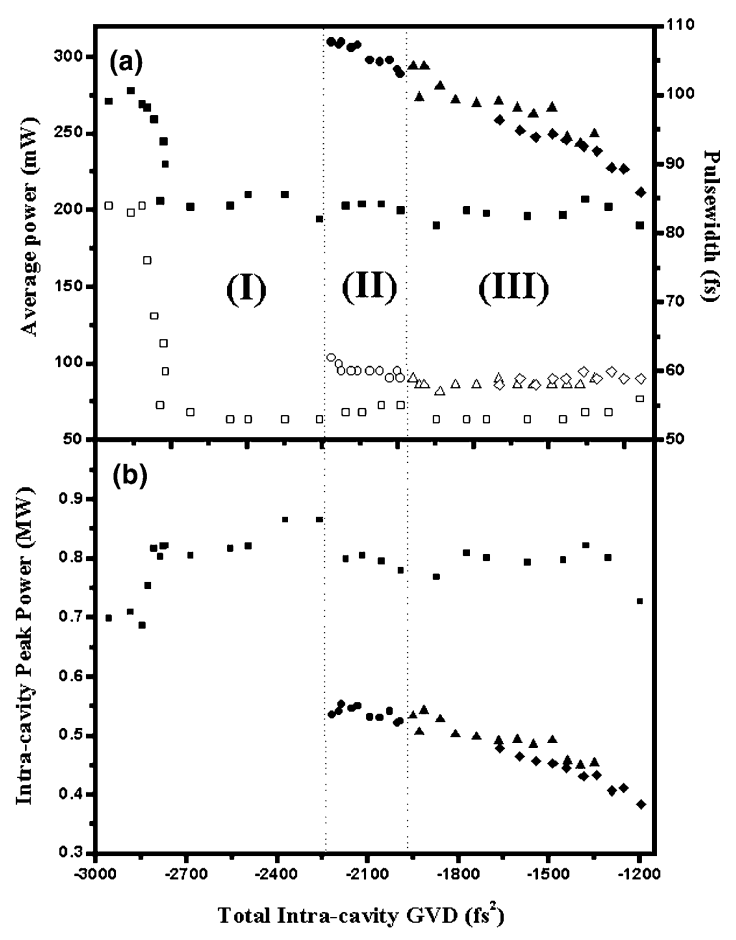

Fig. 6. The average output power (solid symbols) as well as pulsewidth (open symbols) (a) and the intra-cavity peak power; (b) versus the total intra-cavity GVD (or $\beta^{\prime \prime}$ ) for the NML (square), SHML (circle) and pulse-splitting states (triangle and diamond).

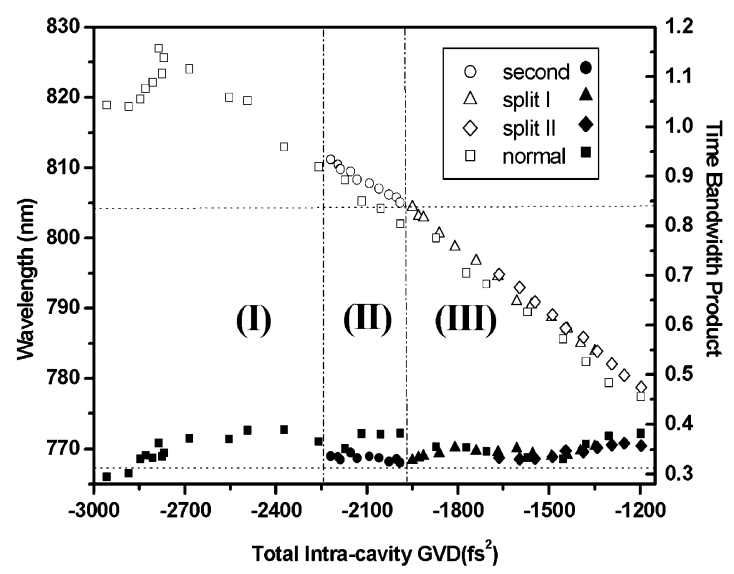

Fig. 7. The wavelength (open symbols) and time bandwidth product (solid symbols) as functions of the total intra-cavity GVD for the NML (square), SHML (circle), and pulse-splitting states (triangle and diamond).
[11], $\mathrm{Cr}^{4+}: \mathrm{YAG}$ [8], and Er/Yb fiber lasers [9] with saturable absorber to explain the observed harmonic mode-locked phenomena.

As the pulse goes through a gain medium, the gain saturation induced group velocity drift may drive the pulse toward the higher gain [10]. Assuming, initially, two pulses per round trip with unequal separation, one of pulses with larger depleted gain will experience larger group-velocity drift relative to the other pulse. After repeating this process in the cavity, two pulses will become equal spacing in one round trip time if the gain recovery time is longer than the cavity round-trip time and the depleted gain is much smaller than the total net gain [10]. Similar analysis can also be applied for $N(N>2)$ pulses to result in equal interpulse spacing among a cavity round trip period or termed $N$ th-harmonic mode locking. Since the recovery time of the Ti:sapphire about $2.5 \mu \mathrm{s}$ is much longer than cavity round trip time of $10.6 \mathrm{~ns}$, the observed harmonic mode locking in our experiment can be explained by the aforementioned gain dynamics.

Two, three, and four pulses with asymmetric ns-spacing were observed similar to that reported in the HAKLM Ti:sapphire laser [6] in which two pulses with temporal separation equal to the round trip time from the laser rod to the output coupler and back will collide in the Kerr medium. Because the two linear arms of our laser system are near equal, if aforementioned pulse colliding mechanism does play the role, the SHML should always be observed and ns-split pulse will not be seen. Furthermore, for the THML, three pulses traveling around the cavity are separated by $1 / 3$ of round trip time $\left(T_{0}\right)$. Assume that one of the pulses is located at the Kerr medium at some moment, the other two pulses will be located ahead and behind of $1 / 3 T_{0}$, they will never collide one another in the active medium. In most cases the interpulse spacing is arbitrary such as that shown in Fig. 4(a) where interpulse spacing is different from the SHML of 5.4 ns or the THML of $3.6 \mathrm{~ns}$. These phenomena observed in our system cannot be explained in terms of pulse colliding within the active medium, and therefore some other mechanisms should be considered in order to explain our results. 
The wavelength dependent gain profile [18] of Ti:sapphire crystal and reflectance of curved mirrors are shown in Fig. 8. We can see that the maximum value of Ti:sapphire gain profile [18] is located around $800 \mathrm{~nm}$ and the high reflection coating of our mirrors has a bandwidth from 780 to $880 \mathrm{~nm}$. The pulses experience the higher instantaneous gain in region II where the operation wavelength is around $802-810 \mathrm{~nm}$. Thus, the dynamic gain introduces enough repulsive force (group velocity drift) to push the ps-split pulses to equal interpulse spacing and form the SHML in region II. However, for multiple pulsing in region III, the operation wavelength of the laser is less than $802 \mathrm{~nm}$ (Fig. 7) so that the loss increases due to abruptly decrease of the reflectance of the mirrors (Fig. 8). This is consistent with experimental observation as shown in region III of Fig. 6 where the average powers of pulses reduce. Assuming two ps-split pulses with slightly different peak intensities, the stronger pulse will experience larger SPM and higher loss than the other.

By introducing an excess loss of the individual pulse into the instantaneous gain to modify the theoretical analysis of gain depletion and recovery model as described in [10], we are able to obtain analytical solution of multiple pulses with unequal spacing occurring in a cavity. The detailed derivation is shown in Appendix A. The difference of interpulse separations in Eq. (A.8) displays an additional term that depends on the loss difference

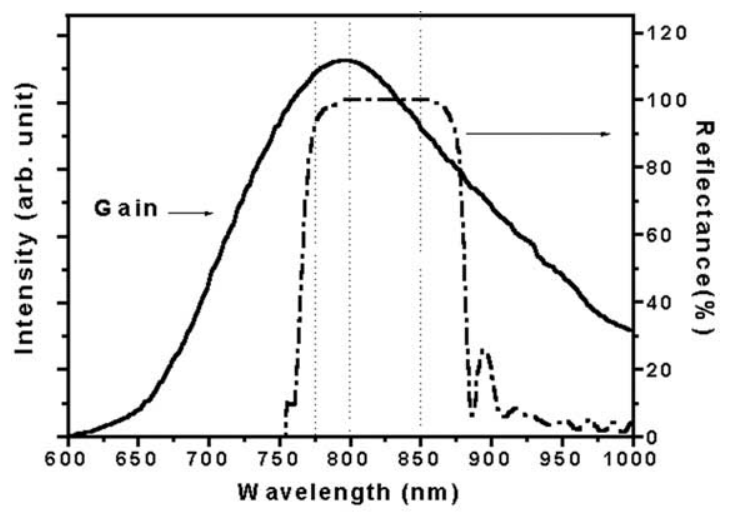

Fig. 8. The gain profile of Ti:sapphire crystal (solid line) and reflectance of curve mirrors (dashed dot line). between pulses as compared with [10, Eq. (12)], where it always converges to zero without the loss difference between pulses. In our system, the round trip loss without considering the excess loss introduced by finite bandwidth of the mirrors is about 3\% such that the instantaneous gain $g_{i}$ is 0.321 in order to balance the loss. For the measured interpulse separations 2.6 and 8.1 ns within a round trip, as shown in Fig. 4(a), which corresponds to 5.4 ns difference of interpulse separations, we calculated from Eq. (A.8) the loss difference between two pulses is only $1 \%$. This value is quite reasonable for the center wavelength below $795 \mathrm{~nm}$ where the mirror reflectance shows strong wavelength dependent (see Fig. 8) to result in ns-pulse-splitting as shown by diamond symbol in Fig. 7. Similar analysis can also be applied for $N$ $(N>2)$ pulses per round trip with equal interpulse spacing to result in the $N$ th-harmonic mode locking or multiple pulses with unequal separation.

\section{Conclusion}

We have observed multiple pulses per round trip occurring in a soft-aperture Kerr-lens modelocked Ti:sapphire laser. By varying the total intra-cavity dispersion, equally spaced harmonic operation and unequally spaced pulses with separation ranging from femtosecond to nanosecond can be found. The harmonic mode locking is observed for the first time to our knowledge without adding saturable absorber that could be reasonably explained by the gain dynamics. An investigation of the laser operated in these regimes in terms of wavelength, bandwidth, pulsewidth, average and peak powers as functions of group velocity dispersion shows that the operating wavelength of unequally spaced pulses is shorter than that of equally spaced pulses. In addition, the information of gain profile for amplifying medium and wavelength-dependent reflectance for mirrors suggest pulses with different wavelength as well as bandwidth may undergo different gain and loss. We then introduce a loss difference of the pulses into gain dynamic analysis and find that the steady state solution of multiple pulses with unequal spacing between the pulses exist in addition to 
harmonic mode locking. The solution expects that only $1 \%$ loss difference is sufficiently to cause 2.6 ns of pulse-splitting.

\section{Acknowledgements}

This work is partially supported by the National Science Council (NSC) and the Ministry of Education of the Republic of China (R.O.C.) under Grants NSC90-2112-009-050, NSC90-2215-E029-001 and 90-E-6-FA-1-4. Mr. Ja-Hon Lin would like to thank NSC for providing fellowship and Mr. Zhi-Cheng Zhan for experimental help.

\section{Appendix A. Gain dynamics including the loss difference}

We consider two pulses in a laser cavity with a fixed round-trip time of $T_{0}$. The interpulse separation of the first pulse to the second pulse is $T_{1}$ and that of the second to the first is $T_{2}$ (note that $T_{1}+T_{2}=T_{0}$ ). The strength of the differential gain across the first pulse is $G_{1}$ and across the second pulse is $G_{2}$. By following the derivative in [10], an analytic description of the interaction dynamics between two pulses is replicated below

$$
\begin{aligned}
& T_{1}(Z+\Delta \mathrm{Z})=T_{1}(Z)+\left[G_{1}(Z)-G_{2}(Z)\right] \frac{\Delta \mathrm{Z}}{2}, \\
& T_{2}(Z+\Delta \mathrm{Z})=T_{2}(Z)-\left[G_{1}(Z)-G_{2}(Z)\right] \frac{\Delta \mathrm{Z}}{2} .
\end{aligned}
$$

These equations describe the relative locations of the pulses as they propagate from a distance $Z$ to $Z+\Delta Z$ with $\Delta Z$ being the round trip distance. Assume the drift of the pulses per round trip time are small and $\Delta \mathrm{Z} \ll 1$, we can convert the difference equations (A.1a) and (A.1b) into the differential equations as

$$
\begin{aligned}
\frac{\mathrm{d} T_{1}}{\mathrm{~d} z} & =\frac{1}{2}\left(G_{1}(Z)-G_{2}(Z)\right), \\
\frac{\mathrm{d} T_{2}}{\mathrm{~d} Z} & =-\frac{1}{2}\left(G_{1}(Z)-G_{2}(Z)\right) .
\end{aligned}
$$

In addition, the instantaneous gain of the two pulses is given by $g_{1}$ and $g_{2}$ with the relation

$g_{2}(Z)=g_{1}(Z)-G_{1}(Z)+\gamma T_{1}(Z)$,

$g_{1}(Z+\Delta Z)=g_{2}(Z)-G_{2}(Z)+\gamma T_{2}(Z)$,

where $\gamma=T_{0} / \tau \ll 1$ and $\tau$ is the normalized recovery time of the gain medium. Now, we assume that the two pulses in the cavity with different wavelengths and linewidths may undergo different losses $l_{i}$ due to wavelength dependent reflectance of laser mirrors. Thus we modify the relation of the time dependent differential gain $G_{i}$ in [10] by adding the extra losses $l_{i}$ to the instantaneous gain $g_{i}$ as

$G_{i}=\frac{1}{\alpha}\left(g_{i}-l_{i}\right)$,

where $\alpha$ is a constant of proportionality which depends upon the pulse energy and magnitude of the total gain. Incorporating this into (A.3a) and (A.3b) yields the iterative equations

$\alpha\left(G_{2}(Z)-G_{1}(Z)\right)+G_{1}(Z)+\left(l_{2}-l_{1}\right)=\gamma T_{1}$,

$\alpha\left(G_{1}(Z+\Delta Z)-G_{2}(Z)\right)+G_{2}(Z)+\left(l_{1}-l_{2}\right)=\gamma T_{2}$.

By adding and subtracting Eqs. (A.5a) and (A.5b), the approximation equation could be obtained by

$$
\begin{aligned}
G_{1}+G_{2}= & \gamma\left(T_{1}+T_{2}\right)=\gamma T_{0}, \\
\left(G_{1}-G_{2}\right)= & -\frac{\gamma}{(2 \alpha-1)}\left(T_{1}-T_{2}\right) \\
& +\frac{2}{(2 \alpha-1)}\left(l_{2}-l_{1}\right),
\end{aligned}
$$

where, Taylor expansion $G_{1}(Z+\Delta Z)=G_{1}(Z)+$ $\Delta Z \mathrm{Zd} G_{1}(Z) / \mathrm{d} Z+\cdots$ is used and the $\mathrm{O}(\Delta \mathrm{Z})$ is dropped since $\Delta \mathrm{Z} \ll 1$. Insert Eqs. (A.6b) into Eqs. (A.2a),(A.2b) yields

$$
\begin{aligned}
\frac{\mathrm{d}}{\mathrm{d} Z}\left(T_{1}-T_{2}\right)= & -\frac{\gamma}{2 \alpha-1}\left(T_{1}-T_{2}\right) \\
& +\frac{2}{2 \alpha-1}\left(l_{2}-l_{1}\right),
\end{aligned}
$$

whose solution is

$$
T_{1}-T_{2}=\frac{2 \Delta l}{\gamma}+\exp \left(\frac{-\gamma}{2 \alpha-1} Z\right) .
$$


This relation describes that the difference of interpulse separations in the cavity will eventually converge to a constant value only determined by the loss difference $\Delta l=l_{2}-l_{1}$. We can see that, if $\Delta l=0$, the only steady-state solution is equally spaced pulses with $T_{1}=T_{2}$ which reduces to the result of [10]. On the contrary, if $\Delta l \neq 0$, the interpulse separation difference $T_{1}-T_{2}=2 \Delta l / \gamma$ is no longer zero that will result in unequal spaced pulses.

\section{References}

[1] C. Spielmann, P.F. Curley, T. Brabec, T. Brabec, F. Krausz, IEEE J. Quantum Electron. 30 (1994) 11001114.

[2] C.-Y. Wang, W. Zhang, K.F. Lee, K.M. Yoo, Opt. Commun. 137 (1997) 89-92.

[3] M. Lai, J. Nicholson, W. Rudolph, Opt. Commun. 142 (1997) 45-49.

[4] M.J. Lederer, B. Luther-Davies, H.H. Tan, C. Jagadish, N.N. Akhmediev, J.M. Soto-Crespo, J. Opt. Soc. B 16 (1999) 895-904.
[5] A.B. Grudinin, D.J. Richardson, D.N. Payne, Electron. Lett. 29 (1993) 1860-1861.

[6] S. Gray, A.B. Grudinin, W.H. Loh, D.N. Payne, Opt. Lett. 20 (1995) 189-191.

[7] A.B. Grudinin, S. Gray, J. Opt. Soc. Am. B 14 (1997) 144-154.

[8] B.C. Collings, K. Bergman, W.H. Knox, Opt. Lett. 22 (1997) 1098-1100.

[9] B.C. Collings, K. Bergman, W.H. Knox, Opt. Lett. 23 (1998) 123-125.

[10] J. Nathan Kutz, B.C. Collings, K. Bergman, W.H. Knox, IEEE J. Quantum Electron. 34 (1998) 1749-1757.

[11] S. Sanders, A. Yariv, J. Paslaski, J.E. Ungar, H.A. Zarem, Appl. Phys. Lett. 58 (1991) 681-683.

[12] K.-H. Lin, W.-F. Hsieh, J. Opt. Soc. Am. B 13 (1996) 1786-1793.

[13] J.K. Ranka, A.L. Gaeta, A. Baltuska, M.S. Pschenichnikov, D.A. Wiersma, Opt. Lett. 22 (1997) 1344-1346.

[14] J.H. Lin, M.D. Wei, W.F. Hsieh, H.H. Wu, J. Opt. Soc. Am. B 18 (2001) 1069-1075.

[15] H.H. Wu, W.F. Hsieh, J. Opt. Soc. Am. B 18 (2001) 7-12.

[16] N.N. Akhmediev, A. Ankiewicz, Opt. Lett. 19 (1994) $545-547$.

[17] N.N. Akhmediev, A. Ankiewicz, J.M. Soto-Crespo, Phy. Rev. Lett. 24 (1997) 4047-4051.

[18] P.F. Moulton, J. Opt. Soc. Am. B 3 (1986) 125-133. 